\title{
Stability of Solid Uranyl Peroxides Under Irradiation
}

Melissa Fairley, ${ }^{\dagger}$ Nicholas M. Myers, ${ }^{\dagger}$ Jennifer E. S. Szymanowski, ${ }^{\ddagger}$ Ginger E. Sigmon, ${ }^{\ddagger}$ Peter C. Burns, ${ }^{\not, l l}$ and Jay A. LaVerne $e^{\dagger,} \S^{*}$

${ }^{\dagger}$ Radiation Laboratory, University of Notre Dame, Notre Dame, IN 46556

tDepartment of Civil and Environmental Engineering and Earth Sciences, University of Notre Dame, Notre Dame, IN 46556

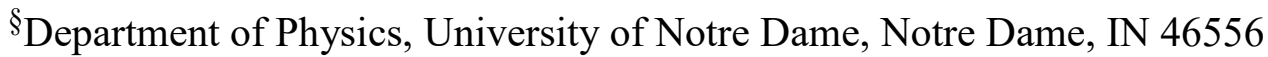

"Department of Chemistry and Biochemistry, University of Notre Dame, Notre Dame, IN 46556

\section{Supporting Information.}

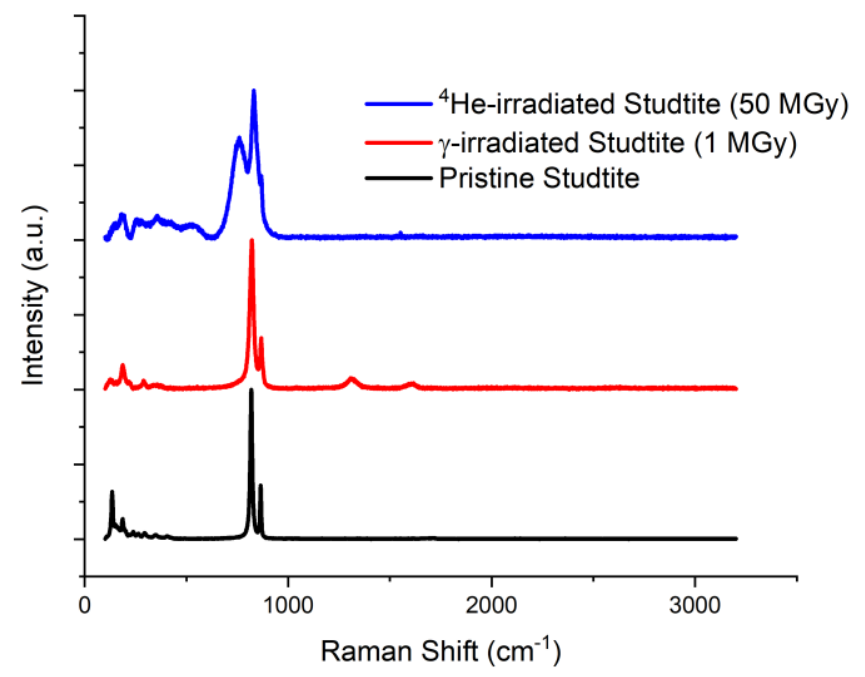

Figure S1. Extended Raman spectra of pristine studtite, $\gamma$-irradiated studtite, and ${ }^{4} \mathrm{He}$-irradiated studtite. 


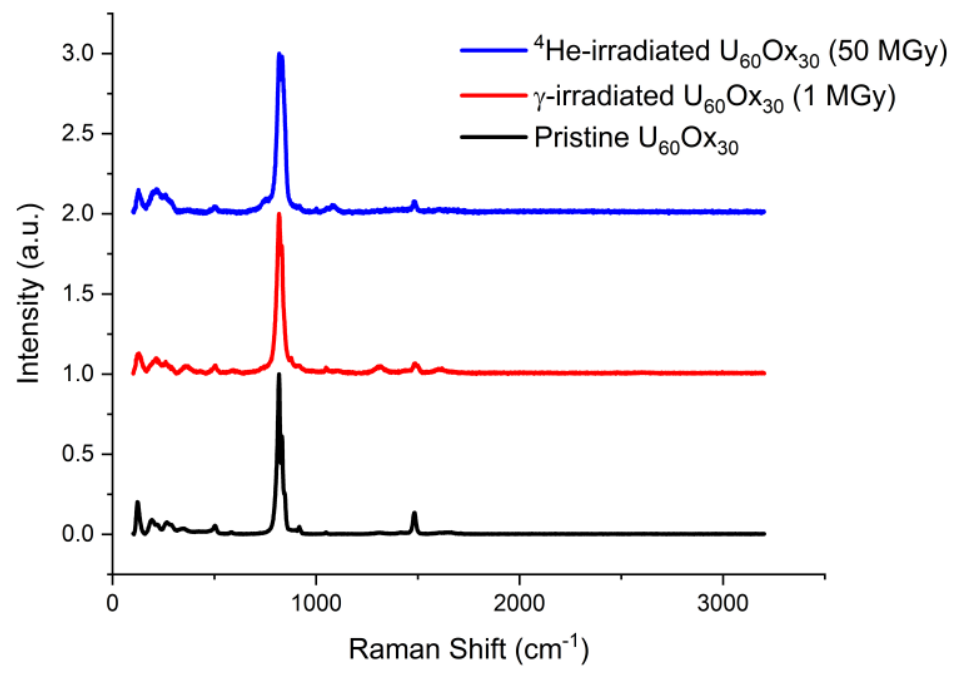

Figure S2. Extended Raman spectra of pristine $\mathrm{U}_{60} \mathrm{Ox}_{30}, \gamma$-irradiated $\mathrm{U}_{60} \mathrm{Ox}_{30}(1 \mathrm{MGy}),{ }^{4} \mathrm{He}-$ irradiated $\mathrm{U}_{60} \mathrm{Ox}_{30}(50 \mathrm{MGy})$.

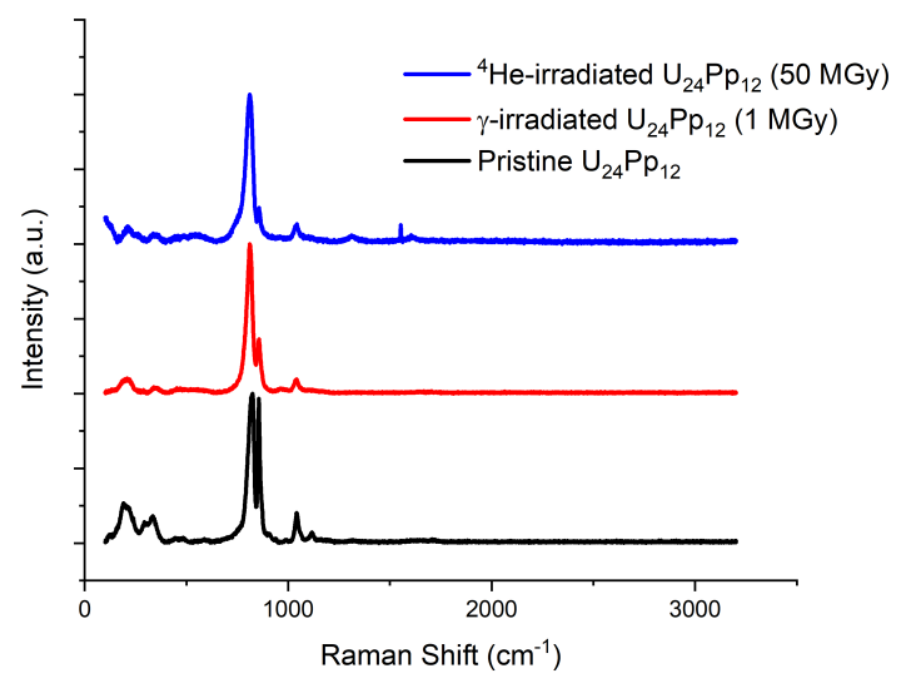

Figure S3. Extended Raman spectra of pristine $\mathrm{U}_{24} \mathrm{Pp}_{12}, \gamma$-irradiated $\mathrm{U}_{24} \mathrm{Pp}_{12}$, and ${ }^{4} \mathrm{He}$-irradiated $\mathrm{U}_{24} \mathrm{Pp}_{12}$. 


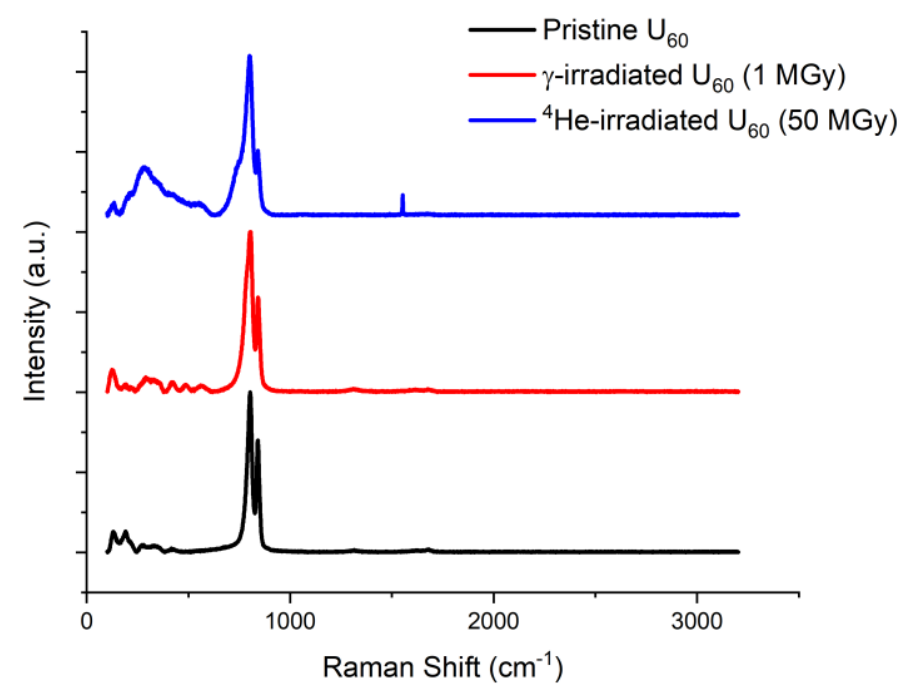

Figure S4. Extended Raman spectra pristine $\mathrm{U}_{60}, \gamma$-irradiated $\mathrm{U}_{60}$, and ${ }^{4} \mathrm{He}$-irradiated $\mathrm{U}_{60}$.
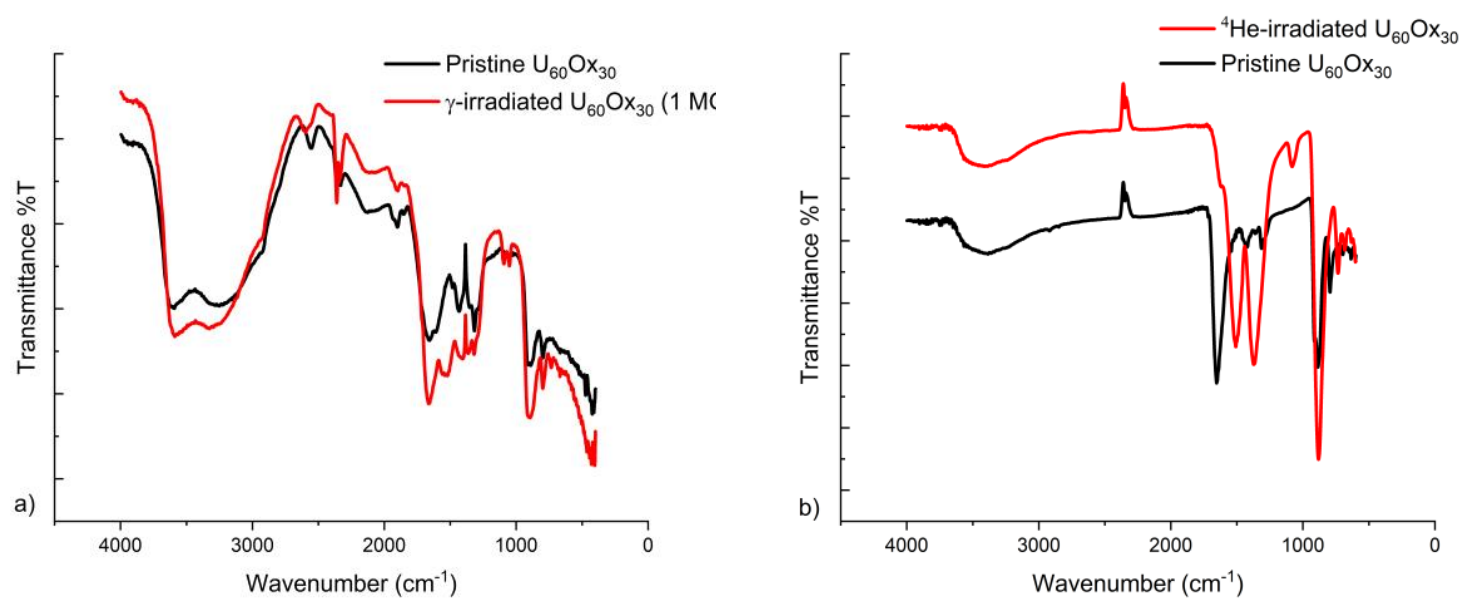

Figure S5. Infrared spectra of $\mathrm{U}_{60} \mathrm{Ox}_{30}$ before and after $1 \mathrm{MGy} \gamma$-irradiation (a) and before and after ${ }^{4} \mathrm{He}$-irradiation (b). 

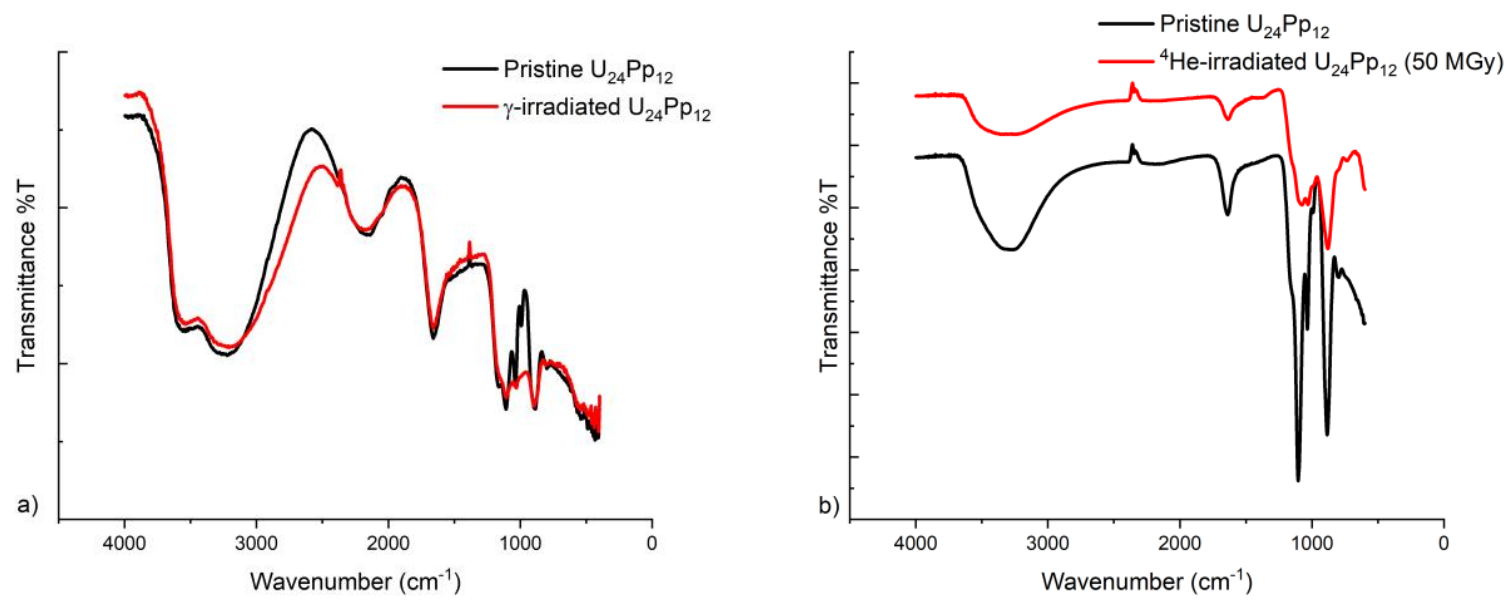

Figure S6. Infrared spectra of $\mathrm{U}_{24} \mathrm{Pp}_{12}$ before and after $1 \mathrm{MGy} \gamma$-irradiation (a) and before and after ${ }^{4} \mathrm{He}$ irradiation (b).
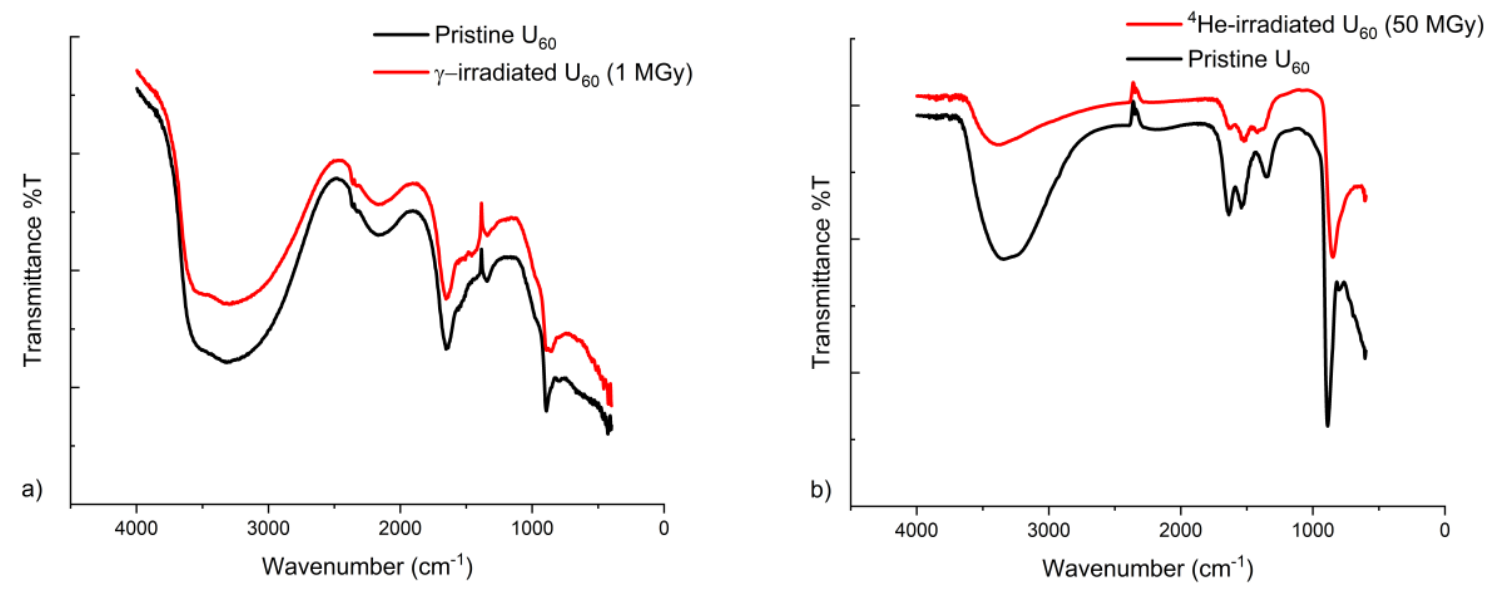

Figure S7. Infrared spectra of $\mathrm{U}_{60}$ before irradiation and after $\gamma$-irradiation (a) and before and after ${ }^{4} \mathrm{He}$-irradiation (b). 


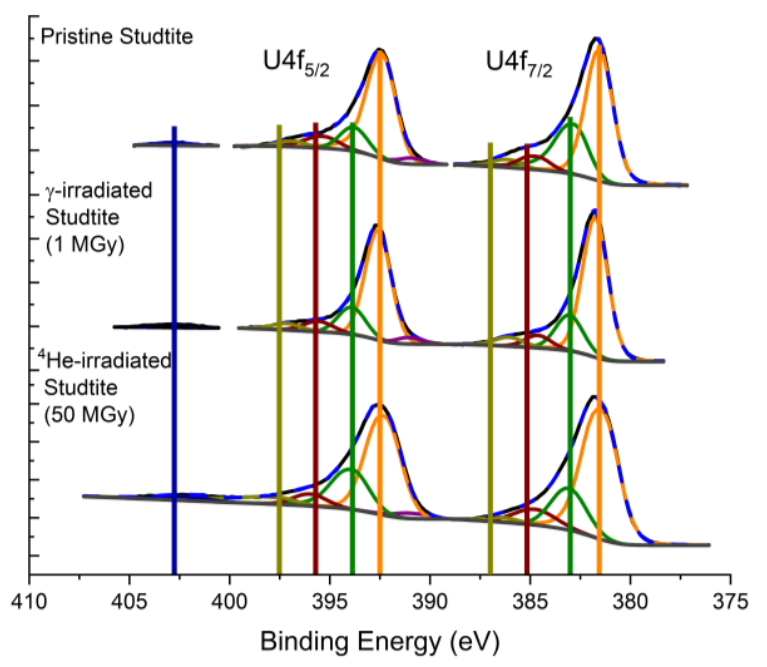

Figure S8. Studtite XPS of $U 4 f_{5 / 2}$ or $U 4^{f / 2}$ envelopes before irradiation, after $\gamma$-irradiation, and after ${ }^{4} \mathrm{He}$-irradiation. The black line is the raw data while the blue dotted line is the fitted sum.

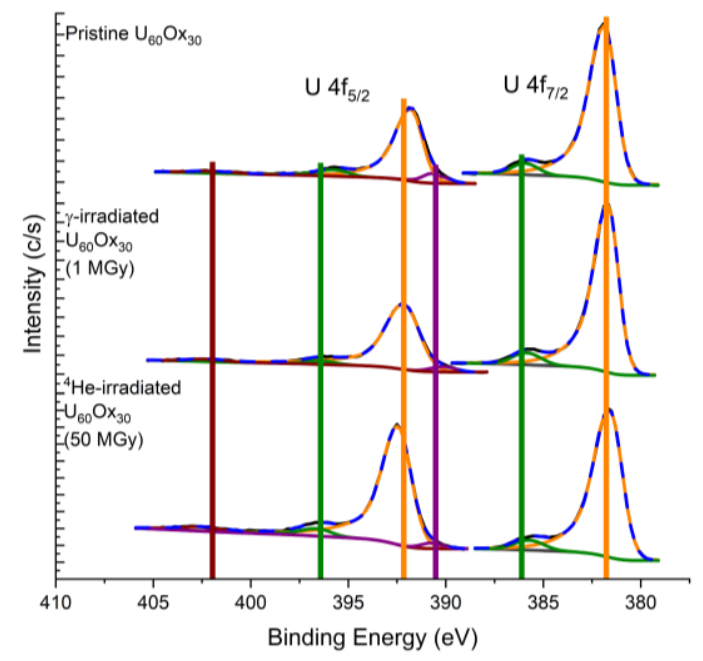

Figure S9. $\mathrm{U}_{60} \mathrm{Ox}_{30} \mathrm{XPS}$ of $\mathrm{U} 4 \mathrm{f}_{5 / 2}$ or $\mathrm{U} 4 \mathrm{f}_{7 / 2}$ envelopes before irradiation, after $\gamma$-irradiation, and after ${ }^{4} \mathrm{He}$-irradiation. The black line is the raw data while the blue dotted line is the fitted sum. 


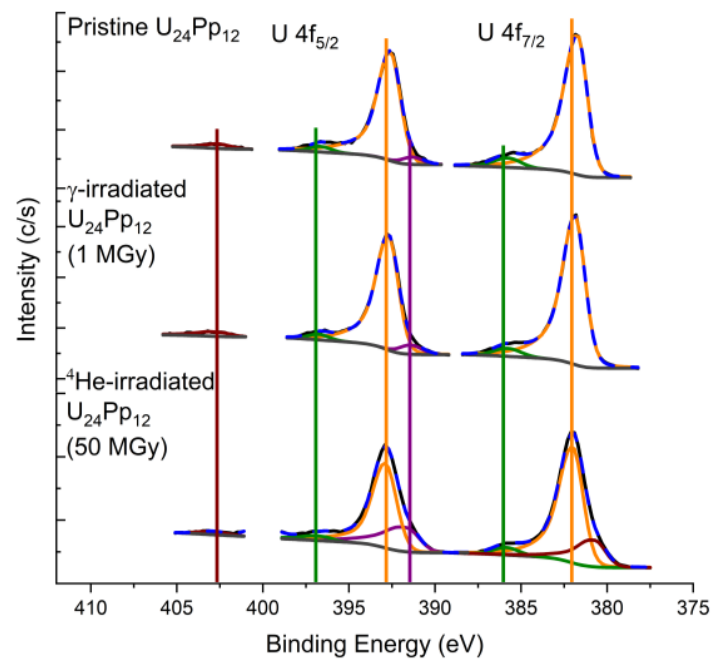

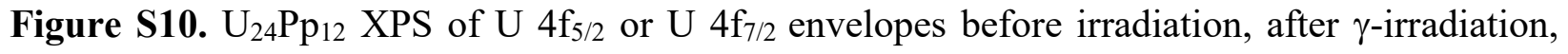
and after ${ }^{4} \mathrm{He}$-irradiation. The black line is the raw data while the blue dotted line is the fitted sum.

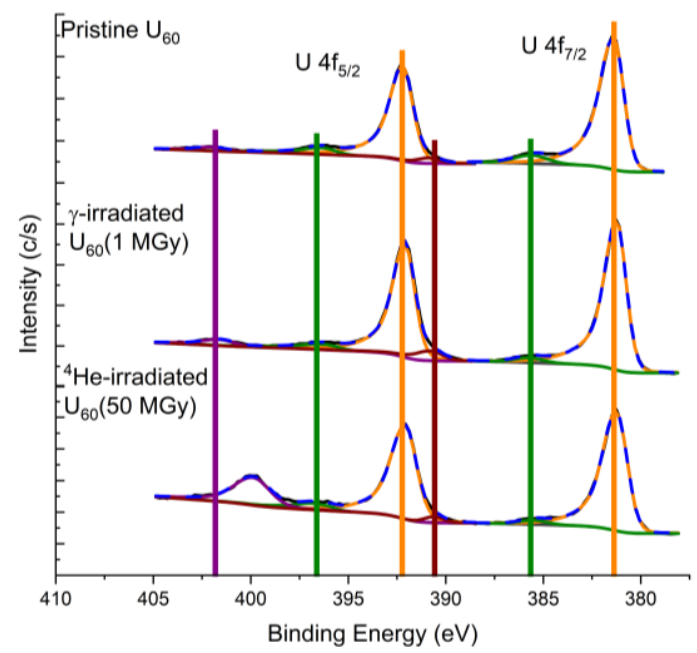

Figure S11. $\mathrm{U}_{60}$ XPS of $\mathrm{U}_{4 \mathrm{f}_{5 / 2}}$ or $\mathrm{U}_{4 \mathrm{f}_{7 / 2}}$ envelopes before irradiation, after $\gamma$-irradiation, and after ${ }^{4} \mathrm{He}$-irradiation. The black line is the raw data while the blue dotted line is the fitted sum. 


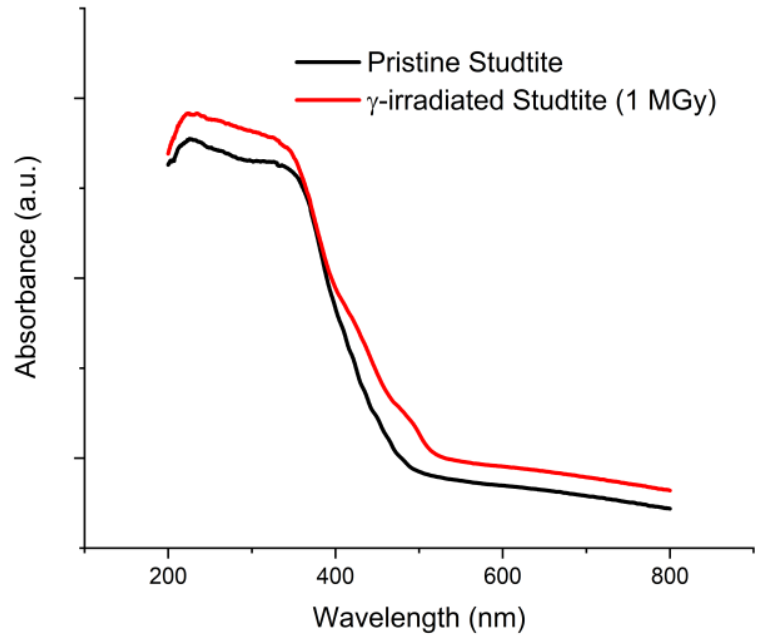

Figure S12. UV-Vis spectra of studtite before and after 1 MGy $\gamma$-irradiation.

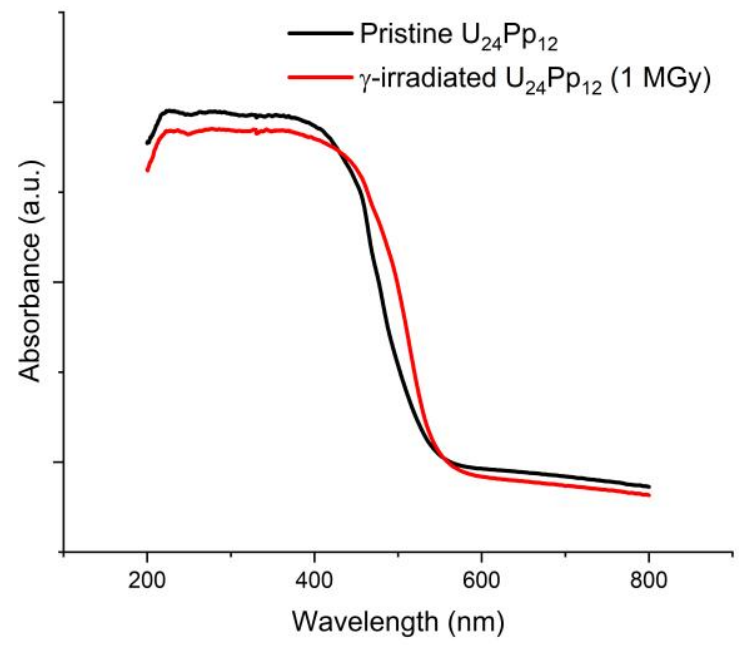

Figure S13. UV-Vis spectra of $\mathrm{U}_{24} \mathrm{Pp}_{12}$ before and after 1 MGy $\gamma$-irradiation. 
a)

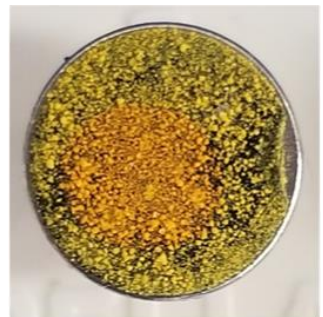

b)
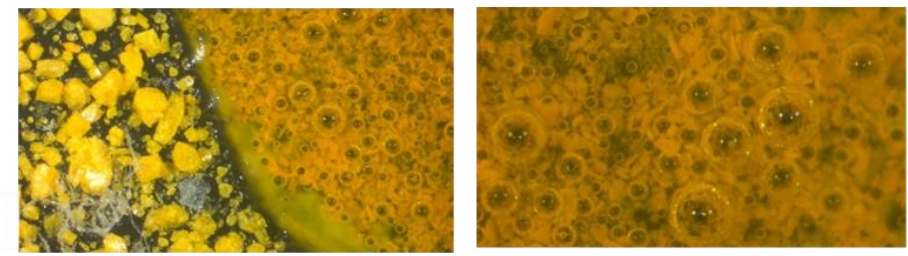

Figure S14. Water reaction with $\mathrm{He}$ ion irradiated $\mathrm{U}_{60}$ prior to addition of water (a) and after addition of water (b).

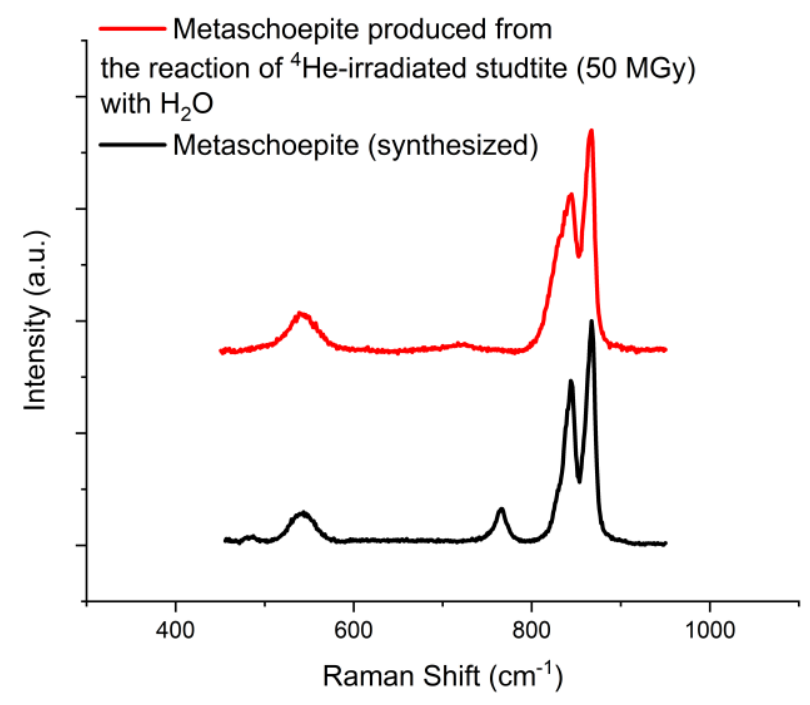

Figure S15. Raman comparison of metaschoepite formed by reacting water with ${ }^{4} \mathrm{He}$-irradiated studtite (50 MGy) and synthesized metaschoepite. 


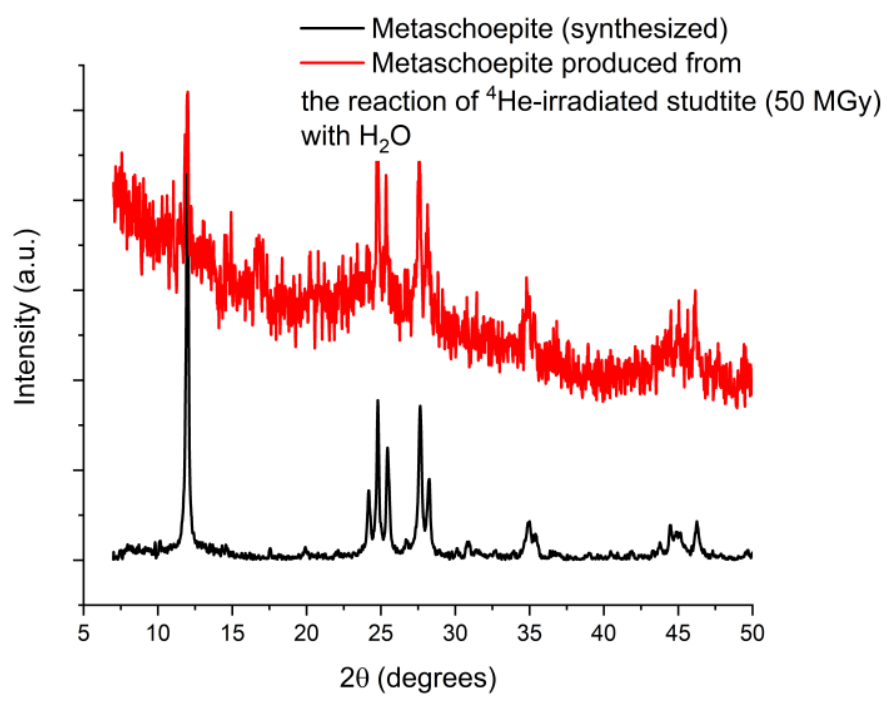

Figure S16. PXRD comparison of metaschoepite formed by reacting water with ${ }^{4} \mathrm{He}$-irradiated studtite (50 MGy) and synthesized metaschoepite.

Table S1. Raman fitting results for studtite

\begin{tabular}{|c|c|c|c|}
\hline Results & Studtite & $\begin{array}{l}\text { Studtite (1 MGy } \gamma \text { - } \\
\text { irradiation) }\end{array}$ & $\begin{array}{l}\text { Studtite (50 MGy } \\
{ }^{4} \text { He-irradiation) }\end{array}$ \\
\hline Iterations & 15 & 13 & 51 \\
\hline Convergence & $1 e-9$ & $1 e-9$ & $1 e-9$ \\
\hline Residual Sum of Squares (RSS) & $9.99705 \mathrm{e} 6$ & $2.67277 \mathrm{e} 6$ & $1.19087 \mathrm{e} 7$ \\
\hline Coefficient of Determination $\left(R^{2}\right)$ & 0.99856 & 0.99479 & 0.99382 \\
\hline Adjusted $\mathrm{R}^{2}$ & 0.99854 & 0.99470 & 0.99366 \\
\hline Peak location $\left(\mathrm{cm}^{-1}\right)$ & $\begin{array}{l}818.51 \pm 0.02 \\
864.51 \pm 0.03\end{array}$ & $\begin{array}{l}820.81 \pm 0.05 \\
867.03 \pm 0.12\end{array}$ & $\begin{array}{l}754.88 \pm 0.29 \\
832.29 \pm 0.16 \\
866.64 \pm 0.38\end{array}$ \\
\hline Full-width half max (FWHM) & $\begin{array}{l}10.84 \pm 0.05 \\
6.04 \pm 0.09\end{array}$ & $\begin{array}{l}18.82 \pm 0.14 \\
9.39 \pm 0.35\end{array}$ & $\begin{array}{l}86.86 \pm 1.18 \\
34.01 \pm 0.66 \\
17.13 \pm 1.38\end{array}$ \\
\hline
\end{tabular}


Table S2. Raman fitting results for $\mathrm{U}_{60} \mathrm{Ox}_{30}$

\begin{tabular}{|c|c|c|c|}
\hline Results & $\mathrm{U}_{60} \mathrm{O} \mathrm{x}_{30}$ & $\begin{array}{l}\mathrm{U}_{60} \mathrm{Ox}_{30}(1 \mathrm{MGy} \gamma- \\
\text { irradiation) }\end{array}$ & $\begin{array}{l}\mathrm{U}_{60} \mathrm{Ox}_{30} \text { (50 MGy } \\
{ }^{4} \mathrm{He} \text {-irradiation) }\end{array}$ \\
\hline Iterations & 36 & 22 & 45 \\
\hline Convergence & $1 e-9$ & $1 e-9$ & $1 e-9$ \\
\hline Residual Sum of Squares (RSS) & $3.21625 \mathrm{e} 7$ & $1.07741 \mathrm{e} 7$ & $2.46635 \mathrm{e} 6$ \\
\hline Coefficient of Determination $\left(R^{2}\right)$ & 0.99161 & 0.99486 & 0.99844 \\
\hline Adjusted $\mathbf{R}^{2}$ & 0.99139 & 0.99473 & 0.99838 \\
\hline Peak location $\left(\mathrm{cm}^{-1}\right)$ & $\begin{array}{l}815.96 \pm 0.07 \\
832.44 \pm 0.11 \\
844.92 \pm 0.29\end{array}$ & $\begin{array}{l}816.61 \pm 0.12 \\
831.71 \pm 0.14 \\
842.66 \pm 0.43\end{array}$ & $\begin{array}{l}746.90 \pm 1.12 \\
816.92 \pm 0.13 \\
833.45 \pm 0.14 \\
844.52 \pm 0.26\end{array}$ \\
\hline Full-width half max (FWHM) & $\begin{array}{l}16.95 \pm 0.24 \\
7.04 \pm 0.41 \\
7.70 \pm 0.94\end{array}$ & $\begin{array}{l}21.64 \pm 0.13 \\
10.63 \pm 0.66 \\
6.15 \pm 1.5\end{array}$ & $\begin{array}{l}33.70 \pm 3.79 \\
23.07 \pm 0.30 \\
15.18 \pm 0.74 \\
14.16 \pm 0.61\end{array}$ \\
\hline
\end{tabular}

Table S3. Raman fitting results for $\mathrm{U}_{24} \mathrm{Pp}_{12}$

\begin{tabular}{|c|c|c|c|}
\hline Results & $U_{24} P p_{12}$ & $\begin{array}{l}\mathrm{U}_{24} \mathrm{Pp}_{12} \text { (1 MGy } \gamma- \\
\text { irradiation) }\end{array}$ & $\begin{array}{l}\mathrm{U}_{24} \mathrm{Pp}_{12} \text { (50 MGy } \\
{ }^{4} \mathrm{He} \text {-irradiation) }\end{array}$ \\
\hline Iterations & 62 & 19 & 28 \\
\hline Convergence & $1 e-9$ & $1 e-9$ & $1 e-9$ \\
\hline Residual Sum of Squares (RSS) & $1.49208 \mathrm{e} 7$ & $6.69851 \mathrm{e} 6$ & $2.8567 \mathrm{e} 6$ \\
\hline Coefficient of Determination $\left(R^{2}\right)$ & 0.98442 & 0.9885 & 0.99509 \\
\hline Adjusted $\mathbf{R}^{2}$ & 0.98402 & 0.9883 & 0.99492 \\
\hline Peak location $\left(\mathrm{cm}^{-1}\right)$ & $\begin{array}{l}808.11 \pm 0.87 \\
820.08 \pm 0.22 \\
857.90 \pm 0.13\end{array}$ & $\begin{array}{l}810.48 \pm 0.11 \\
857.35 \pm 0.26\end{array}$ & $\begin{array}{l}758.46 \pm 3.68 \\
803.55 \pm 0.89 \\
818.93 \pm 0.37 \\
856.07 \pm 0.21\end{array}$ \\
\hline Full-width half max (FWHM) & $\begin{array}{l}24.74 \pm 1.31 \\
13.34 \pm 0.81 \\
10.81 \pm 0.40\end{array}$ & $\begin{array}{l}29.28 \pm 0.37 \\
13.83 \pm 0.81\end{array}$ & $\begin{array}{l}65.48 \pm 8.75 \\
34.39 \pm 2.04 \\
20.70 \pm 1.42 \\
9.43 \pm 0.69\end{array}$ \\
\hline
\end{tabular}


Table S4. Raman fitting results for $\mathrm{U}_{60}$

\begin{tabular}{|c|l|l|l|}
\hline \multicolumn{1}{|c|}{ Results } & $\mathbf{U}_{\mathbf{6 0}}$ & $\begin{array}{l}\mathbf{U}_{\mathbf{6 0}} \text { (1 MGy } \\
\gamma \text {-irradiation) }\end{array}$ & $\begin{array}{l}\mathbf{U}_{\mathbf{6 0}} \text { (50 MGy }{ }^{\mathbf{4}} \mathbf{H e}- \\
\text { irradiation) }\end{array}$ \\
\hline Iterations & 18 & 21 & 126 \\
\hline Convergence & $1 \mathrm{e}-9$ & $1 \mathrm{e}-9$ & $1 \mathrm{e}-9$ \\
\hline Residual Sum of Squares (RSS) & $6.11918 \mathrm{e} 7$ & $1.14717 \mathrm{e}$ & $1.98000 \mathrm{e}$ \\
\hline Coefficient of Determination $\mathbf{R}^{\mathbf{2}}$ ) & 0.98686 & 0.99371 & 0.99582 \\
\hline Adjusted $\mathbf{R}^{\mathbf{2}}$ & 0.98663 & 0.99354 & 0.99571 \\
\hline Peak location $\mathbf{( c m}^{-\mathbf{1}} \mathbf{)}$ & $800.91 \pm 0.11$, & $785.63 \pm 0.48$, & $748.45 \pm 0.99$, \\
& $841.76 \pm 0.12$ & $804.69 \pm 0.17$, & $799.74 \pm 0.13$, \\
& & $843.03 \pm 0.11$ & $843.43 \pm 0.27$ \\
\hline Full-width half max (FWHM) & $25.18 \pm 0.37$, & $30.75 \pm 1.00$, & $92.78 \pm 2.84$, \\
& $12.38 \pm 0.37$ & $19.84 \pm 0.57$, & $36.65 \pm 0.58$, \\
& & $12.74 \pm 0.35$ & $9.97 \pm 0.88$ \\
\hline
\end{tabular}

